\title{
Probability density functions for advective-reactive transport in radial flow
}

\author{
S. Broyda $\cdot$ M. Dentz $\cdot$ D. M. Tartakovsky
}

Published online: 27 April 2010

(c) The Author(s) 2010. This article is published with open access at Springerlink.com

\begin{abstract}
We study transport of a reactive solute in a chemically heterogeneous porous medium whose chemical properties are uncertain. The dissolved substance undergoes a heterogeneous chemical reaction with a solid phase in the presence of advection caused by extraction/injection from a point source. We present semi-analytical solutions for the probability density function of the solute concentration, which allows us to quantify predictive uncertainty associated with uncertain reaction rate constants for both linear and nonlinear reactions. This enables one to compute probabilities of rare events, which are required for quantitative risk analyses.
\end{abstract}

Keywords Uncertainty quantification - Geochemistry · Heterogeneity $\cdot$ Stochastic modeling

\section{Introduction}

Subsurface heterogeneity and the ubiquitous lack of sufficient site parameterizations are some of the key factors that render predictions of subsurface flow and transport inherently uncertain. This predictive uncertainty is often quantified by treating relevant flow and transport parameters as

S. Broyda · D. M. Tartakovsky ( $)$

Department of Mechanical and Aerospace Engineering, University of California, San Diego, 9500 Gilman Drive, Mail Code 0411, La Jolla, CA 92093, USA

e-mail:dmt@ucsd.edu

\section{Dentz}

Institute of Environmental Assessment and Water Research (IDÆA-CSIC), Barcelona, Spain

e-mail: marco.dentz@idaea.csic.es random fields and corresponding governing equations as stochastic. The state-of-the-art of the field of stochastic hydrogeology and its future have been the subject of the August 2004 special issue of Stochastic Environmental Research \& Risk Assessment (e.g., Neuman 2004; Dagan 2004).

Monte Carlo simulations (MCS) are currently a dominant approach to solving stochastic partial differential equations (SPDEs) of subsurface hydrology. While conceptually straightforward, MCS have a number of potential drawbacks. For nonlinear transient three-dimensional phenomena, e.g., reactive transport which is the focus of the present study, MCS lack well-established convergence criteria and often prove to be computationally prohibitive due to high CPU and storage demands. Numerical approaches, such as stochastic finite element methods based on polynomial chaos expansions or stochastic collocation methods, provide a viable alternative to MCS but under certain conditions their computational burden might exceed that of MCS (e.g., Xiu and Tartakovsky 2006, Sect. 3.3.3). MCS and its numerical alternatives provide little or no physical insight into computed moments of system states.

Another alternative to MCS relies on (deterministic) moment differential equations (MDEs) to describe spatiotemporal evolution of ensemble moments of the system states. Equations for the first ensemble moments, e.g., mean hydraulic head and mean concentration, are alternatively referred to as averaged, effective, or upscaled equations (Christakos 2003; Zavala-Sanchez et al. 2007; Neuman and Tartakovsky 2009). Equations for the second ensemble moments, e.g., (co)variances of hydraulic head and concentrations, serve to quantify uncertainty associated with predictions based on upscaled models (Neuman 1993; Cushman et al. 1995; Dagan and Cvetkovic 1996). 
Derivations of MDEs typically require a closure approximation (e.g., perturbation expansions) that limit their range of applicability. Their applications to nonlinear phenomena entail linearization of the reactive terms that often compromises the robustness and accuracy of resulting solutions. Finally, MDEs cannot be used to predict rare events, which are described by the tails of statistical distributions and lie at the foundation of probabilistic risk analyses (Tartakovsky 2007; Winter and Tartakovsky 2008; Bolster et al. 2009).

One approach to computing a full (single-point) statistical distribution, e.g., probability density function (PDF), of a system state is to assume its functional form and parameterize the latter (e.g., find the mean and variance of a Gaussian PDF) by "solving" corresponding SPDEs. Examples of such "parametric solutions" include assumed Gaussian PDF for hydraulic head in steady-state and transient saturated flows (Nachabe and Morel-Seytoux 1995) and pressure in steady-state (Amir and Neuman 2001) and transient (Amir and Neuman 2004) unsaturated flow, and $\beta$-distributions for concentration of conservative (Bellin and Tonina 2007) and reactive (Cirpka et al. 2008) solutes migrating in groundwater flow. While promising, such approaches are akin to guessing a solution of a differential equation, except the validity of assumed PDFs as solutions of SPDEs cannot be verified by direct substitution and requires, instead, extensive numerical testing. In general, the validity of an assumed PDF depends on statistical properties of input parameters, initial and boundary conditions, etc. in a manner that cannot be ascertained a priori. For example, Nachabe and Morel-Seytoux (1995) showed that hydraulic head in saturated flows can be assigned a Gaussian distribution if the correlation length of hydraulic conductivity is small, and Tartakovsky and Guadagnini (2001) and Tartakovsky et al. (2003) demonstrated that a Gaussian approximation of pressure head in unsaturated flows is valid if the variance of saturated hydraulic conductivity is small.

PDF methods overcome many of the shortcomings discussed above by deriving a deterministic equation for the PDF of a system state. First developed for application in turbulence and combustion (Pope 2000, Chapter 12; and the references therein), PDF methods were introduced into subsurface hydrology by Shvidler (1985)—see Shvidler and Karasaki (2003) as a more accessible reference-to derive the PDF of the concentration of a conservative solute that is being advected by a randomly fluctuating velocity field. Applications of the PDF methods to reactive transport in porous media can be found in Lichtner and Tartakovsky (2003) and Tartakovsky et al. (2009). One of the advantages of PDF methods is their ability to treat reactive terms exactly, without resorting to linearization.

While turbulent combustion is typically characterized by homogeneous chemical reactions with known (deterministic) reaction rates, reactive transport in porous media often involves heterogeneous reactions with uncertain (random) reaction rates. Thus, the context of subsurface reactive transport requires the development of new methods and approaches. Lichtner and Tartakovsky (2003) made the first step in this direction by analyzing the effects of uncertain reaction rate "constants" on uncertainty in predictions of solute concentration in a reactive batch, i.e., in the absence of flow. Tartakovsky et al. (2009) extended their analysis by allowing for the presence of deterministic (i.e., known with certainty) flow velocity. Computational examples in that analysis dealt with constant velocity fields. The main goal of the present study is to elucidate the effects of spatial, albeit deterministic, variability of flow velocity on the predictive uncertainty in advective-reactive transport phenomena.

Section 2 contains a mathematical model of reactive transport to a pumping well in macroscopically homogeneous aquifers with uncertain reaction rate coefficients $k(\mathbf{x})$. In Sect. 3, we derive a solution for the concentration PDF in terms of an integral of the random field $k(\mathbf{x})$. An algorithm for computing the PDF of this stochastic integral is described in Sect. 4. In Sect. 5, we provide a computational example for a solute undergoing a second-order heterogeneous reaction.

\section{Problem formulation}

Consider advective transport of a reactive solute to a well that operates at a constant pumping rate $Q$ in a confined macroscopically homogeneous aquifer of infinite lateral extent and thickness $b$, whose transmissivity is $T$ and porosity is $\phi$. Drawdown $s(r)$ at a distance $r$ from the well satisfies the Laplace equation subject to the mass conservation at the well of small radius $r_{w}$,

$\nabla^{2} s=0, \quad \lim _{r_{\omega} \rightarrow 0} 2 \pi r_{\omega} T \frac{\partial s}{\partial r}\left(r_{\omega}\right)=-Q$,

$s(r=\infty)=0$

This results in macroscopic flow velocity $\mathbf{v}=(v, 0)$, with the radial component $v=-T /(\phi b) \partial s / \partial r$ given by the Thiem solution

$v(r)=\frac{Q}{2 \pi \phi b r}$.

While being advected by the velocity field $\mathbf{v}$, the solute undergoes a heterogeneous chemical reaction between a dissolved species $\mathcal{C}$ and a solid $\mathcal{C}_{(s)}$,

$\alpha \mathcal{C} \rightleftharpoons \mathcal{C}_{(s)}$,

where $\alpha$ is the stoichiometric coefficient. The speed with which $c$, the concentration of $\mathcal{C}$, reaches its equilibrium 
level $c_{\mathrm{eq}}$ is determined from the product of the laboratory measured kinetic rate constant $k_{0}\left[\mathrm{~mol} \mathrm{~L}^{-2} \mathrm{~T}^{-1}\right]$ for reaction (3) and specific surface area $A\left[\mathrm{~L}^{-1}\right]$ of a porous matrix, designated as $k$ and referred to as the kinetic rate constant

$k=k_{0} A c_{\mathrm{eq}}^{-\alpha}$.

In this definition, the kinetic rate constant $k\left[\left(\mathrm{~mol} \mathrm{~L}^{-3}\right)^{1-}\right.$ ${ }^{\alpha} \mathrm{T}^{-1}$ ] includes the contribution from the specific surface area $A$, assumed to be constant in time in the following. On the local scale $\omega$, the evolution of the solute concentration $c(\mathbf{x}, t)$ in steady macroscopic velocity field $\mathbf{v}(\mathbf{x})$ can be described by an advection-reaction equation (ARE)

$\frac{\partial c}{\partial t}+\nabla \cdot(\mathbf{v} c)=-\frac{k}{\phi} f_{\alpha}(c), \quad f_{\alpha}(c) \equiv \alpha\left(c^{\alpha}-c_{\mathrm{eq}}^{\alpha}\right)$,

where $f_{\alpha}(c)$ represents the product of the stoichiometric coefficient and the affinity factor giving the degree of disequilibrium. The transport equation is subject to the initial condition

$c(\mathbf{x}, t=0)=c_{\text {in }}(\mathbf{x})$

and appropriate boundary conditions. The initial concentration $c_{\text {in }}$ may be larger or smaller than the equilibrium concentration $c_{\mathrm{eq}}$ resulting in precipitation of the reacting species or dissolution of the solid matrix, respectively. It is worthwhile emphasizing that the methodology presented below is equally applicable to other types of chemical reactions and other functional forms of $f_{\alpha}(c)$.

The following assumptions underlie the problem formulation (2)-(6).

1. In the spirit of the analyses by Neuman (1993), Shvidler and Karasaki (2003), Tartakovsky et al. (2009) and many others, we neglect dispersion on the local scale $\omega$. In this view, dispersion is an emerging transport phenomenon that manifests itself on scales larger than $\omega$. This assumption is valid for transport phenomena in which mixing in the aqueous phase does not control reactions.

2. Both the porosity $\phi$ and the reaction rate constant $k$ do not change with time. This assumption implies that equilibrium concentration is reached before appreciable changes in porous matrix occur.

3. The ARE (5) holds on a mesoscale $\omega$, which is much larger than the pore scale. It breaks down when the reaction rate $f_{\alpha}(c)$ becomes large enough to produce gradients on the scale of a single pore (Battiato et al. 2009), a phenomenon that can be captured with either pore-scale (Tartakovsky et al. 2007) or hybrid (Tartakovsky et al. 2008) simulations.

\subsection{Formulation of a probabilistic model}

Since the kinetic reaction rate constant $k$ in (4) depends on the specific surface area $A$ of a porous medium, it is determined by a pore- (or grain-) size distribution that can be highly variable even if the medium in question is macroscopically homogeneous. In a typical example relevant to the present analysis, a (constant) value of hydraulic conductivity $K$ is determined from a pumping test by using, say, the Thiem solution of (1) to interpret the data. The specific surface area $A$ within the support volume of the pumping test can be expected to vary significantly, rendering the reaction rate constant $k(\mathbf{x})$ variable and highly uncertain. In general, many reactive processes are controlled by pore-scale heterogeneity that is harder to characterize with certainty than continuum-scale heterogeneity that controls flow. Consequently, we focus on quantification of uncertainty in the reaction rate constant $k(\mathbf{x})$ by treating it as a random field, while allowing flow velocity $\mathbf{v}(\mathbf{x})$ to vary in space deterministically in accordance with (2).

To be concrete, we treat the reaction rate constant $k(\mathbf{x})$ as a statistically homogeneous (second-order stationary) multi-variate lognormal random field (Lichtner and Tartakovsky 2003, and the references therein),

$p_{k}(k)=\frac{1}{k \sqrt{2 \pi} \sigma} \exp \left[-\frac{(\ln k-\mu)^{2}}{2 \sigma^{2}}\right]$,

with a two-point correlation function $\rho\left(\mathbf{x}_{1}, \mathbf{x}_{2}\right) \equiv \rho_{k}(d)$ that depends on the distance $d=\left|\mathbf{x}_{1}-\mathbf{x}_{2}\right|$ between points $\mathbf{x}_{1}$ and $\mathbf{x}_{2}$ and has a correlation length $l_{k}$. Here the constants $\mu$ and $\sigma^{2}$ are the mean and variance of the random field $\ln k(\mathbf{x})$, respectively. They are related to the mean, $\langle k\rangle$, and variance, $\sigma_{k}^{2}$, of the reaction rate constant $k(\mathbf{x})$ by

$\mu=\ln \langle k\rangle-\frac{1}{2} \ln \left(1+\frac{\sigma_{k}^{2}}{\langle k\rangle^{2}}\right) \quad$ and $\quad \sigma^{2}=\ln \left(1+\frac{\sigma_{k}^{2}}{\langle k\rangle^{2}}\right)$.

The initial and equilibrium distributions $c_{0}$ and $c_{\mathrm{eq}}$ in general can be either certain (deterministic) or uncertain (random). For simplicity, in this study we treat them as deterministic, although extensions to the random case are straightforward.

\subsection{Non-dimensional form}

Let $t_{a}$ denote the advection time scale defined as the time it takes a solute to travel one correlation length $l_{k}$ with the characteristic macroscopic velocity $U$, 
$t_{a}=\frac{l_{k}}{U}, \quad U=\frac{Q}{2 \pi \phi b}$.

Let $t_{r}$ denote the reaction time scale defined by

$t_{r}=\frac{\phi}{\langle k\rangle} c_{\mathrm{eq}}^{1-\alpha}$.

Then the relative importance of these transport mechanisms can be quantified in terms of the dimensionless Damköhler number $D a=t_{a} / t_{r}$ as

$D a=\frac{2 \pi b}{Q} l_{k}\langle k\rangle c_{\mathrm{eq}}^{\alpha-1}$.

Introducing dimensionless variables

$$
\begin{aligned}
\hat{\mathbf{x}} \equiv \frac{\mathbf{x}}{l_{k}}, \quad \hat{t} \equiv \frac{t}{t_{a}}, \quad \hat{k} \equiv \frac{k}{\langle k\rangle}, \quad \hat{\mathbf{v}} \equiv \frac{\mathbf{v}}{U}, \quad \hat{c} \equiv \frac{c}{c_{\mathrm{eq}}}, \\
\hat{c}_{\mathrm{in}} \equiv \frac{c_{\mathrm{in}}}{c_{\mathrm{eq}}}
\end{aligned}
$$

and accounting for the incompressibility of the macroscopic flow velocity $\mathbf{v}(\mathbf{x})$ in (2), we transform (5) and (6) into their dimensionless form

$\frac{\partial \hat{c}}{\partial \hat{t}}+\hat{\mathbf{v}} \cdot \hat{\nabla} \hat{c}=-D a \hat{k} \hat{f}_{\alpha}(\hat{c}), \quad \hat{f}_{\alpha}(\hat{c})=\alpha\left(\hat{c}^{\alpha}-1\right)$,

$\hat{c}(\hat{\mathbf{x}}, \hat{t}=0)=\hat{c}_{\text {in }}(\hat{\mathbf{x}})$.

It follows from (9) to (11) that the dimensionless advection time scale is $\hat{t}_{a} \equiv t_{a} / t_{a}=1$ and the dimensionless reaction time scale is $\hat{t}_{r} \equiv t_{r} / t_{a}=D a^{-1}$.

To relate the statistics of the dimensionless reaction rate constant $\hat{k}(\mathbf{x})$ to those of its dimensional counterpart $k(\mathbf{x})$, we employ Reynolds decomposition of the random field $k(\mathbf{x})$ into its ensemble mean $\langle k\rangle$ and zero-mean fluctuations $k^{\prime}(\mathbf{x})$. In dimensionless form, this gives

$\hat{k}(\mathbf{x})=1+\hat{k}^{\prime}(\mathbf{x}), \quad \hat{k}^{\prime}(\mathbf{x})=\frac{k^{\prime}(\mathbf{x})}{\langle k\rangle}, \quad\left\langle k^{\prime}(\mathbf{x})\right\rangle=0$.

It follows from (14) that $\langle\hat{k}\rangle=1$ and $\hat{\sigma}_{k}^{2}=\sigma_{k}^{2} /\langle k\rangle^{2}$. The single-point PDF of log-normal $\hat{k}(\mathbf{x})$ takes the form

$p_{\hat{k}}(k)=\frac{1}{k \sqrt{2 \pi} \hat{\sigma}} \exp \left[-\frac{(\ln k-\hat{\mu})^{2}}{2 \hat{\sigma}^{2}}\right]$,

where, as can be seen from (8),

$\hat{\mu}=-\frac{1}{2} \ln \left(1+\hat{\sigma}_{k}^{2}\right) \quad$ and $\quad \hat{\sigma}^{2}=\ln \left(1+\hat{\sigma}_{k}^{2}\right)$.

The two-point correlation function of $\hat{k}(\mathbf{x})$ takes the form

$\rho_{\hat{k}}(\hat{d})=\rho_{k}(\hat{d}), \quad \hat{d} \equiv \frac{d}{l}$.

It is related to $\rho(\hat{d})$, the dimensionless two-point correlation function of $\ln \hat{k}(\hat{\mathbf{x}})$, by the relationship $\rho_{\hat{k}}(\hat{d})=\frac{\mathrm{e}^{\rho(\hat{\mathrm{d}}) \theta^{2}}-1}{\mathrm{e}^{\hat{\sigma}^{2}}-1}$.

Note that the dimensionless reaction rate constant $\hat{k}(\mathbf{x})$ has a unit correlation length.

In the following, we will drop the hat $\hat{*}$ to simplify the notation.

\section{Probability density function of solute concentration}

Parametric uncertainty in the reactive transport model (13) is quantified by treating the reaction rate constant $k(\mathbf{x})$ as a random field with given statistics, (15)-(17). Solving SPDE (13) propagates this uncertainty through the modeling process, with predictive uncertainty expressed in terms of the PDF of concentration. Our goal is to derive an exact relationship between PDF of the kinetic rate constant and unknown PDF of concentration $c(\mathbf{x}, t)$.

Along the family of characteristics $\mathbf{x}(t)$ defined by

$\frac{\mathrm{d} \mathbf{x}}{\mathrm{d} t}=\mathbf{v}(\mathbf{x}), \quad \mathbf{x}(0)=\boldsymbol{\xi}$,

where $\xi$ "labels" a characteristic by its point of origin at time $t=0$, concentration $c(\mathbf{x}, t)$ in (13) evolves according to a stochastic ordinary differential equation

$\frac{\mathrm{dc}}{\mathrm{d} t}=-\operatorname{Dak}[\mathbf{x}(t)] f_{\alpha}(c), \quad c(0)=c_{\mathrm{in}}(\boldsymbol{\xi})$.

An implicit solution of (20) can be written as (Tartakovsky et al. 2009)

$\mathcal{F}_{\alpha}(c)=-\operatorname{DaK}(t), \quad \mathcal{K}(t) \equiv \int_{0}^{t} k\left[\mathbf{x}\left(t^{\prime}\right)\right] \mathrm{d} t^{\prime}$,

where $\mathcal{F}_{\alpha}$ is given by a linear combination of hypergeometric functions ${ }_{2} F_{1}(a, b, c ; d)$,

$$
\begin{aligned}
\mathcal{F}_{\alpha}(c) \equiv & \frac{c}{\alpha}{ }_{2} F_{1}\left(\frac{1}{\alpha}, 1,1+\frac{1}{\alpha} ; c^{\alpha}\right) \\
& -\frac{c_{\text {in }}(\xi)}{\alpha}{ }_{2} F_{1}\left(\frac{1}{\alpha}, 1,1+\frac{1}{\alpha} ; c_{i n}^{\alpha}\right) .
\end{aligned}
$$

For a given velocity field $\mathbf{v}(\mathbf{x}),(21)$ and (19) provide a mapping between the integral of $k(\mathbf{x})$ and $c(\mathbf{x}, t)$. Combined with the probability invariance under a change of variables, $p_{\mathcal{K}}(K ; t) \mathrm{d} K=p_{c}(c ; \mathbf{x}, t) \mathrm{d} c$, this mapping allows one to express the PDF of $c, p_{c}(c ; \mathbf{x}, t)$, in terms of the PDF of $\mathcal{K}$, $p_{\mathcal{K}}(K ; t)$, as

$p_{c}(c ; \mathbf{x}, t)=\frac{1}{D a f_{\alpha}(c)} p_{\mathcal{K}}\left[-\frac{1}{D a} \mathcal{F}_{\alpha}(c) ; t\right]$.

Substituting the dimensionless form of (2) into (19) written in the polar coordinates $\mathbf{x}=(r, \theta)^{T}$, we obtain 
$\frac{\mathrm{dr}}{\mathrm{d} t}=\frac{1}{r}, \quad r(0)=\xi_{r} ; \quad \frac{\mathrm{d} \theta}{\mathrm{d} t}=0, \quad \theta(0)=\xi_{\theta}$

where $\boldsymbol{\xi}=\left(\xi_{r}, \xi_{\theta}\right)^{T}$. This gives a family of characteristics $\mathbf{x}(t)=[r(t), \theta]^{T}$ with

$r(t)=\sqrt{\xi_{r}^{2}+2 t}, \quad \theta=\xi_{\theta}$.

The statistical homogeneity of the random field $k[\mathbf{x}(t)]$ implies that for a fixed $\mathbf{x}=(r, \theta)$ the random field $k\left(\sqrt{r^{2}-2 t}, \theta\right)$ is statistically equivalent to $k\left(\sqrt{r^{2}-2 t}, 0\right)$. Thus, in the following we identify

$k[\mathbf{x}(t)] \equiv k\left(\sqrt{r^{2}-2 t}\right)$.

It then follows from (17) that the two-point correlation function of $k^{\prime}(r, t)$ for a fixed point in space is

$$
\begin{aligned}
& \left\langle k^{\prime}\left(\sqrt{r^{2}-2 t}, 0\right) k^{\prime}\left(\sqrt{r^{2}-2 t^{\prime}}, 0\right)\right\rangle \\
& \quad=\sigma_{k}^{2} \rho_{k}\left(\sqrt{r^{2}-2 t}-\sqrt{r^{2}-2 t^{\prime}}\right) .
\end{aligned}
$$

It remains to compute the PDF of the random function $\mathcal{K}(t)$ in (21) along the characteristics (25) in terms of the PDF of the kinetic rate constant $k(t)$.

\section{Computing PDF of $\mathcal{K}(t)$}

It follows from (21) and (27) that the mean and variance of $\mathcal{K}(t)$ are given by

$$
\langle\mathcal{K}(t)\rangle=t
$$

and

$\sigma_{\mathcal{K}}^{2}(t)=\sigma_{k}^{2} \int_{0}^{t} \int_{0}^{t} \rho_{k}\left(\sqrt{r^{2}-2 t^{\prime}}-\sqrt{r^{2}-2 t^{\prime \prime}}\right) \mathrm{d} t^{\prime} \mathrm{d} t^{\prime \prime}$.

If the dimensionless time $t \ll r^{2}$, then $\sqrt{r^{2}-2 t} \approx r-t / r$ and (29) can be simplified to give

$$
\begin{aligned}
\sigma_{\mathcal{K}}^{2}(t) & =\sigma_{k}^{2} r^{2} \int_{0}^{t / r} \int_{0}^{t / r} \rho_{k}\left(t^{\prime}-t^{\prime \prime}\right) \mathrm{d} t^{\prime} \mathrm{d} t^{\prime \prime} \\
& =2 \sigma_{k}^{2} r^{2} \int_{0}^{t / r}\left(\frac{t}{r}-t^{\prime}\right) \rho_{k}\left(t^{\prime}\right) \mathrm{d} t^{\prime} .
\end{aligned}
$$

To compute $p_{\mathcal{K}}(K ; t)$, the single-point PDF of $\mathcal{K}(t)$ in (21), we note that a change of the variable of integration transforms the integral

$\mathcal{K}(t)=\int_{0}^{t} k\left(\sqrt{r^{2}-2 t^{\prime}}\right) \mathrm{d} t^{\prime}$

into
$\mathcal{K}(t)=\int_{\sqrt{r^{2}-2 t}}^{r} \xi k(\xi) \mathrm{d} \xi$.

Discretizing $L \equiv r-\sqrt{r^{2}-2 t}$, the length of the interval of integration in (32), into $N$ sub-intervals of equal length $L / N$ yields

$\mathcal{K}(t) \approx \frac{L}{N} \sum_{i=0}^{N-1} \xi_{i} k\left(\xi_{i}\right)$

$\xi_{i}=r+i \frac{L}{N}, \quad i=0, \ldots, N-1$.

Next, we write the single-point $\operatorname{PDF}$ of $\mathcal{K}(t)$ as

$p_{\mathcal{K}}(K ; t)=\langle\delta[K-\mathcal{K}(t)]\rangle \approx \frac{1}{R} \sum_{j=1}^{R} \delta\left[K-\{\mathcal{K}(t)\}_{j}\right]$,

where the angular brackets denote the average over all possible realizations of the correlated random field $k(\mathbf{x})$, and the second equality represents its approximation computed with $R$ realizations. Substituting (33) into (34), we obtain a numerical approximation of the PDF of $\mathcal{K}(t)$,

$p_{\mathcal{K}}(K ; t)=\frac{1}{R} \sum_{j=1}^{R} \delta\left[K-\frac{L}{N} \sum_{i=0}^{N-1} \xi_{i} k^{(j)}\left(\xi_{i}\right)\right]$,

where $k^{(j)}\left(\xi_{i}\right)$ denotes the $j$-th realization of the ensemble of random series $\left\{k\left(\xi_{i}\right)\right\}$.

Figure 1 shows the PDF of $\mathcal{K}(t)$ computed from (35) with $R=10^{5}$ realizations of the correlated lognormal field $k(\xi)$ with $\sigma=1$. (Recall that the dimensionless $k$ has unit mean and unit correlation length.) The PDF is evaluated at dimensionless distance $r=100$ for several values of dimensionless time $t$. One can see that as dimensionless time $t$ increases, the PDF of $\mathcal{K}(t)$ approaches a Gaussian distribution. This behavior is to be expected from the central limit theorem.

To verify the accuracy of this solution, we compare the first and second moments computed from (33) with their exact counterparts (28) and (29). The results of this comparison are presented in Table 1 in terms of the relative error $\mathcal{E}_{\mathcal{A}}=\left|\mathcal{A}_{\mathrm{ex}}-\mathcal{A}_{\mathrm{ap}}\right| /\left|\mathcal{A}_{\mathrm{ex}}\right|$, where $\mathcal{A}=\langle\mathcal{K}\rangle$ or $\sigma_{\mathcal{K}}^{2}$, and $\mathcal{A}_{\text {ex }}$ and $\mathcal{A}_{\text {ap }}$ denote the exact and approximate values of $\mathcal{A}$, respectively. Since in the simulations reported in Fig. 1 we discretized the interval $L(t)$ into the same number of subintervals $N=100$ for all $t$, the relative error increase with time, reflecting the decreasing accuracy of the quadrature approximation (33). This errors can be reduced by increasing $N$ as $t$ increases.

As dimensionless times $t$ increases, $L$ becomes large and eventually can be discretized into sub-intervals of unit length, $L / N=1$. Since the dimensionless correlation length of $k$ is $\hat{l}=1$, random variables $k(r+i L / N)$ with different $i$ 's might be treated as independent from each other. This 

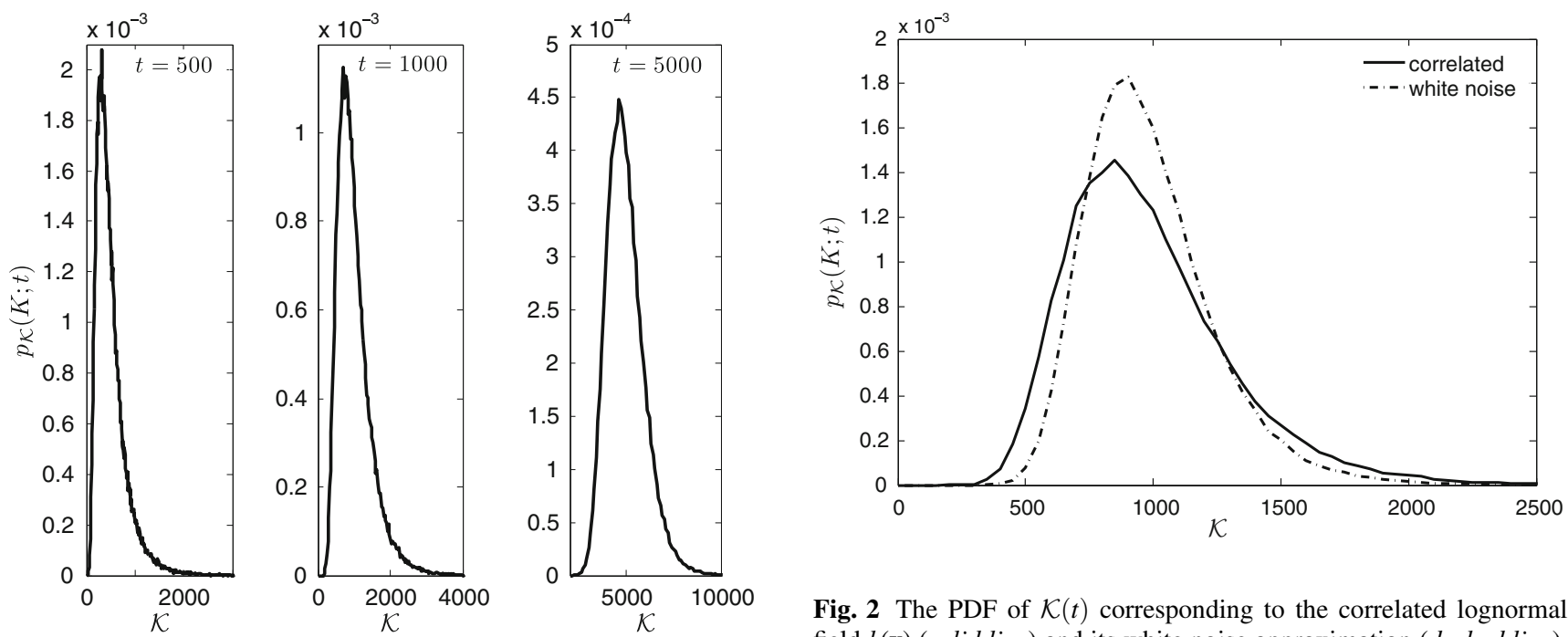

Fig. 1 Snapshots of temporal evolution of the PDF of $\mathcal{K}(t)$ at $r=100$ for $\sigma=1$

Table 1 Relative errors of the first and second moments of $\mathcal{K}(t)$ computed at $r=100$ for $\sigma=1$

\begin{tabular}{lll}
\hline & \multicolumn{2}{l}{ Relative errors in } \\
\cline { 2 - 3 } & Mean & Variance \\
\hline$t=500$ & 0.0001 & 0.0053 \\
$t=1000$ & 0.0009 & 0.0067 \\
$t=5000$ & 0.0097 & 0.1409 \\
\hline
\end{tabular}

white-noise approximation of the random field $k$ was used by Tartakovsky et al. (2009) to replace the correlation function of $k$ with the Dirac delta function $\left\langle k^{\prime}\left(t_{1}\right) k^{\prime}\left(t_{2}\right)\right\rangle=$ $\sigma_{k}^{2} \delta\left(t_{1}-t_{2}\right)$. For spatially varying velocity fields $\mathbf{v}(\mathbf{x})$, this approximation holds only for relatively large $L$, i.e., large dimensionless time $t$, and even then it introduces significant errors (Fig. 2). The impact of the white-noise approximation on the PDF of concentration is investigated below.

\section{Computational example}

Let us consider the non-equilibrium behavior of a reacting solute and its relaxation to equilibrium. The simulation results below are for heterogeneous nonlinear reactions with the stochiometric coefficient $\alpha=2$, in which case (22) reduces to

$$
\mathcal{F}_{2}(c)=\frac{1}{2} \operatorname{artanh} c_{\text {in }}(\xi)-\frac{1}{2} \operatorname{artanh} c .
$$

We set the initial concentration at the position $\xi$ to $c_{\text {in }}=0$, so that the simulations below correspond to dissolution of a solid matrix and result in a reactive system wherein the dimensionless concentration $c(\mathbf{x}, t) \leq 1$. The Damköhler

Fig. 2 The PDF of $\mathcal{K}(t)$ corresponding to the correlated lognormal field $k(\mathbf{x})$ (solid line) and its white noise approximation (dashed line). In both cases, $r=50, t=1000$, and $\sigma=1$

number is set to $D a=10^{-3}$, and the dimensionless variance of $\ln k$ is set to $\sigma=1$. Finally, we assume an exponential correlation function from $(18), \rho(d)=\exp (-d)$.

Figure 3 exhibits the temporal evolution of the PDF of concentration at point $r=50$. As time increases, the dimensionless concentration $c$ evolves from the deterministic initial concentration $c_{\text {in }}=0$ to the deterministic equilibrium concentration $c_{\mathrm{eq}}=1$. Correspondingly, the single-point PDF of $c$ varies from the delta function $p(c ; \mathbf{x}, 0)=\delta(c)$ at $t=0$ to the delta function $p(c ; \mathbf{x}, 0)=\delta(c-1)$ for large $t$. Between these deterministic limits, the uncertainty in predictions of $c$ increases, which manifests itself in the widening of the PDF of $c$.

Analyzing the temporal evolution of the concentration PDF in uniform flow, Tartakovsky et al. (2009) found that it can be adequately approximated by treating $k^{\prime}(\xi)$ as a Gaussian white noise, and that the concentration PDF is

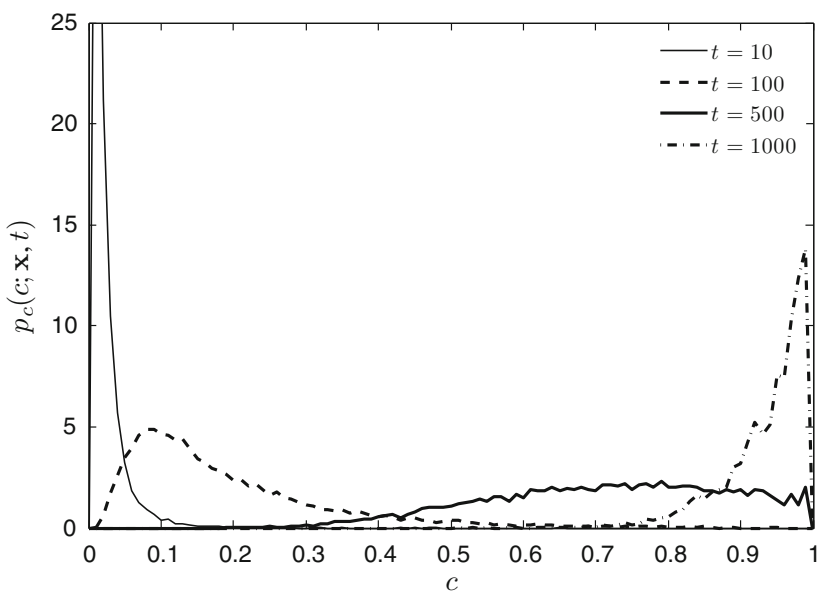

Fig. 3 Snapshots of temporal evolution of the PDF of concentration $p_{c}(c ; \mathbf{x}, t)$ at $r=50$ 
approximately Gaussian for $\sigma=1$ (see their Fig. 2a). The flow non-uniformity renders the white-noise approximation of $k[\mathbf{x}(t)]$ in the stochastic integral (32) problematic (Fig. 2), except in the limit of large $r$ and $t$ and even then this approximation introduces significant errors. Figure 4 suggests the impact of this approximation on the concentration PDF is more pervasive in non-uniform flows and that the concentration PDFs exhibit long tails.

Figure 5 demonstrates that the shape of the concentration PDF and, thus, predictive uncertainty are significantly affected by the Damköhler number $D a$. As the Damköhler number (i.e., the ratio between the advective and reactive time scales) increases, the concentration reaches equilibrium, and the concentration PDF approach the delta function $p_{c} \sim \delta(c-1)$, faster. Recall that dimensionless time is defined relative to the advection time scale, so that reaction time scales as $D a^{-1}$.

\section{Summary and conclusions}

We analyzed advective-reactive transport in chemically heterogeneous porous media with uncertain parameters. Radial flow is induced by extraction/injection from a point source, and a dissolved substance undergoes a non-linear heterogeneous chemical reaction with the solid phase. We developed an efficient stochastic approach to map a distribution of the spatially varying reaction rate constant onto a single point PDF of the concentration of the reactive solute.

We found that the shape and evolution of the PDF of the concentration of reactive solutes are significantly affected by both spatial distributions of the advective velocity and the Damköhler number. This makes the reliance on assumed PDFs problematic.

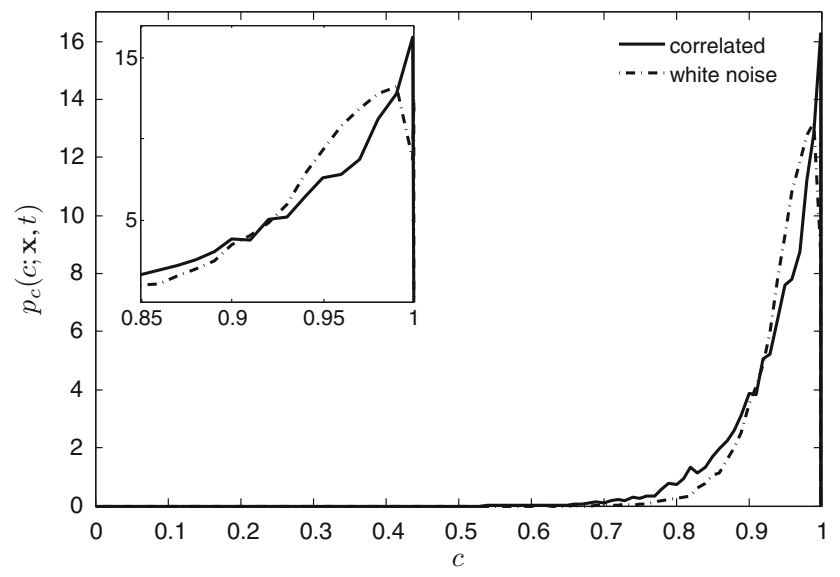

Fig. 4 The PDF of concentration $p_{c}(c ; \mathbf{x}, t)$ at $r=50$ and $t=1000$. The solid and dashed lines correspond to the correlated and uncorrelated lognormal fields $k(\mathbf{x})$, respectively. In these simulations, we set $N=2 \times 10^{5}$

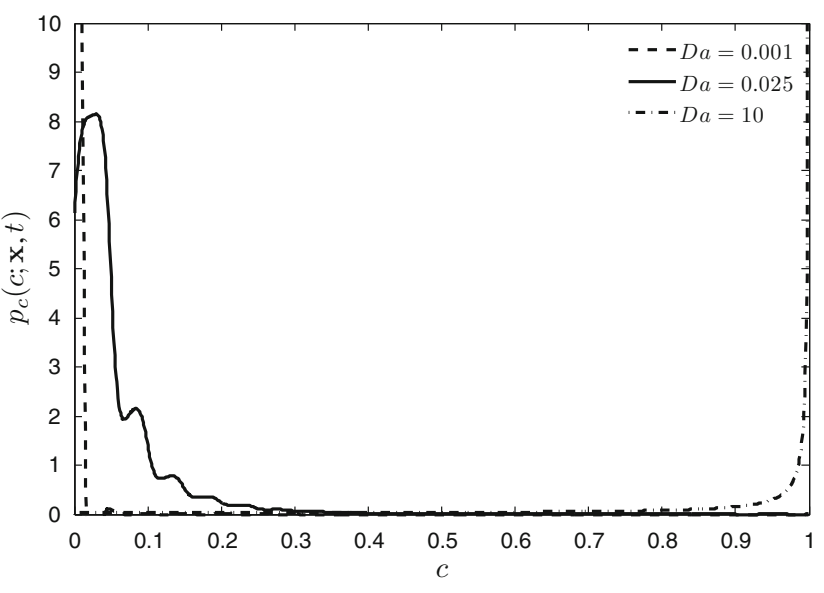

Fig. 5 Snapshots of temporal evolution of the PDF of concentration $p_{c}(c ; \mathbf{x}, t)$ at $r=100, t=1$ for different Damköhler numbers

In future studies, we will analyze the combined effects of uncertainty in hydraulic conductivity (flow velocity) and reaction rate coefficients. Effects of molecular diffusion and local-scale hydrodynamic dispersion are likewise left for future investigations.

Acknowledgements This research was supported by the DOE Office of Science Advanced Scientific Computing Research (ASCR) program in Applied Mathematical Sciences, and by the Office of Science (BER), Cooperative Agreement No. DE-FC02-07ER64324.

Open Access This article is distributed under the terms of the Creative Commons Attribution Noncommercial License which permits any noncommercial use, distribution, and reproduction in any medium, provided the original author(s) and source are credited.

\section{References}

Amir O, Neuman SP (2001) Gaussian closure of one-dimensional unsaturated flow in randomly heterogeneous soils. Transp Porous Media 44(2):355-383

Amir O, Neuman SP (2004) Gaussian closure of transient unsaturated flow in random soils. Transp Porous Media 54(1):55-77

Battiato I, Tartakovsky DM, Tartakovsky AM, Scheibe T (2009) On breakdown of macroscopic models of mixing-controlled heterogeneous reactions in porous media. Adv Water Resour 32: 1664-1673. doi:10.1016/j.advwatres.2009.08.008

Bellin A, Tonina D (2007) Probability density function of nonreactive solute concentration in heterogeneous porous formations. J Contam Hydrol 94:109-125

Bolster D, Barahona M, Dentz M, Fernandez-Garcia D, Sanchez-Vila X, Trinchero P, Valhondo C, Tartakovsky DM (2009) Probabilistic risk analysis of groundwater remediation strategies. Water Resour Res 45:W06,413. doi:10.1029/2008WR007551

Christakos G (2003) Another look at the conceptual fundamentals of porous media upscaling. Stoch Environ Res Risk Assess 17(5):276-290. doi:10.1007/s00477-003-0150-8

Cirpka OA, Schwede RL, Luo J, Dentz M (2008) Concentration statistics for mixing-controlled reactive transport in random heterogeneous media. J Contam Hydrol 98(1-2):61-74

Cushman JH, Hu BX, Deng FW (1995) Nonlocal reactive transport with physical and chemical heterogeneity: localization errors. Water Resour Res 31(9):2219-2237 
Dagan G (2004) On application of stochastic modeling of groundwater flow and transport. Stoch Environ Res Risk Assess 18(4):266-267. doi:10.1007/s00477-004-0191-7

Dagan G, Cvetkovic V (1996) Reactive transport and immiscible flow in geological media. I. General theory. Proc Roy Soc Lond A 452:285-301

Lichtner PC, Tartakovsky DM (2003) Upscaled effective rate constant for heterogeneous reactions. Stoch Environ Res Risk Assess 17(6):419-429

Nachabe MH, Morel-Seytoux HJ (1995) Perturbation and Gaussian methods for stochastic flow problems. Adv Water Resour 18(1):1-8. doi:10.1016/0309-1708(94)00023-X

Neuman SP (1993) Eulerian-Lagrangian theory of transport in spacetime nonstationary velocity fields: exact nonlocal formalism by conditional moments and weak approximation. Water Resour Res 29(3):633-645

Neuman SP (2004) Stochastic groundwater models in practice. Stoch Environ Res Risk Assess 18(4):268-270. doi:10.1007/s00477004-0192-6

Neuman SP, Tartakovsky DM (2009) Perspective on theories of anomalous transport in heterogeneous media. Adv Water Resour 32(5):670-680. doi:10.1016/j.advwatres.2008.08.005

Pope SB (2000) Turbulent flows. Cambridge University Press, Cambridge, UK

Shvidler M, Karasaki K (2003) Probability density functions for solute transport in random field. Transp Porous Media 50(3): 243-266

Shvidler MI (1985) Statistical hydrodynamics of porous media [in Russian]. Nedra, Moscow, USSR
Tartakovsky AM, Meakin P, Scheibe TD, West RME (2007) Simulation of reactive transport and precipitation with smoothed particle hydrodynamics. J Comp Phys 222:654-672

Tartakovsky AM, Tartakovsky DM, Scheibe TD, Meakin P (2008) Hybrid simulations of reaction-diffusion systems in porous media. SIAM J Sci Comput 30(6):2799-2816

Tartakovsky DM (2007) Probabilistic risk analysis in subsurface hydrology. Geophys Res Lett 34:L05,404. doi:10.1029/2007 GL029245

Tartakovsky DM, Guadagnini A (2001) Prior mapping for nonlinear flows in random environments. Phys Rev E 64:5302(R)5305(R). doi:10.1103/PhysRevE.64.035302

Tartakovsky DM, Guadagnini A, Riva M (2003) Stochastic averaging of nonlinear flows in heterogeneous porous media. J Fluid Mech 492:47-62. doi:10.1017/S002211200300538X

Tartakovsky DM, Dentz M, Lichtner PC (2009) Probability density functions for advective-reactive transport in porous media with uncertain reaction rates. Water Resour Res 45:W07,414. doi: 10.1029/2008WR007383

Winter CL, Tartakovsky DM (2008) A reduced complexity model for probabilistic risk assessment of groundwater contamination. Water Resour Res 44:W06,501. doi:10.1029/2007WR006599

Xiu D, Tartakovsky DM (2006) Numerical methods for differential equations in random domains. SIAM J Sci Comput 28(3): $1167-1185$

Zavala-Sanchez V, Dentz M, Sanchez-Vila X (2007) Effective dispersion in a chemically heterogeneous medium under temporally fluctuating flow conditions. Adv Water Resour 30(5): 1342-1354. doi:10.1016/j.advwatres.2006.11.010 